\title{
ON HAMILTONIAN CIRCUITS IN FINITE GRAPHS
}

\author{
C. ST. J. A. NASH-WILlIAMS
}

Let $G$ be a finite graph with $n(\geqq 3)$ vertices and no loops or multiple edges. Two vertices are adjacent if they are joined by an edge. The degree of a vertex $v$ will be denoted by $d(v)$. A way is an alternating sequence of distinct vertices and edges of $G$ in which each pair of successive terms are incident and the first and last terms are vertices. The $i$ th vertex of a way $W$ will be denoted by $w_{i}$. A circuit is obtained from a way with more than two vertices whose first and last terms are adjacent by adding the edge joining them. The number of edges in a way or circuit is its length. A circuit of length $n$ is Hamiltonian. Pósa [1] proved the following interesting theorem.

Suppose that $G$ satisfies the following conditions:

(i) for every positive integer $k$ less than $\frac{1}{2}(n-1)$, the number of vertices of degree not exceeding $k$ is less than $k$,

(ii) the number of vertices of degree not exceeding $\frac{1}{2}(n-1)$ is less than or equal to $\frac{1}{2}(n-1)$.

Then $G$ has a Hamiltonian circuit.

(We remark that Condition (ii) is contained in Condition (i) if $n$ is even.)

This note presents a slightly different proof of Pósa's theorem, which avoids the construction of additional graphs.

Suppose that $G$ satisfies (i) and (ii). If a component of $G$ has $r$ vertices, the degrees of these vertices cannot exceed $r-1$ and therefore $r>\frac{1}{2} n$ by (i). Therefore each component of $G$ has more than $\frac{1}{2} n$ vertices and so $G$ must be connected. Let $m$ be the maximum of the lengths of the ways in $G$. Choose a way $W$ of length $m$ such that $d\left(w_{1}\right)+d\left(w_{m+1}\right)$ is as large as possible. Let $S$ be the set of all vertices $w_{i}$ such that $w_{1}$ is adjacent to $w_{i+1}$. We note that $w_{m+1} \notin S$. Since there is no way of length $m+1$ in $G, w_{1}$ is not adjacent to any vertex not in $W$, and hence is adjacent to $d\left(w_{1}\right)$ terms of $W$. Therefore $S$ has cardinal number $d\left(w_{1}\right)$. Moreover, if $w_{i} \in S$, then

$$
w_{i}, w_{i-1}, \cdots, w_{1}, w_{i+1}, w_{i+2}, \cdots, w_{m+1}
$$

are the vertices of a way of length $m$, and therefore $d\left(w_{i}\right) \leqq d\left(w_{1}\right)$ by the manner in which $W$ was chosen. Hence the degrees of the $d\left(w_{1}\right)$ elements of $S$ do not exceed $d\left(w_{1}\right)$ and therefore by (i), $d\left(w_{1}\right) \geqq \frac{1}{2}(n-1)$.

Received by the editors January 29, 1965. 
By a similar argument, $d\left(w_{m+1}\right) \geqq \frac{1}{2}(n-1)$. Moreover, if $d\left(w_{1}\right)$ and $d\left(w_{m+1}\right)$ were both $\frac{1}{2}(n-1)$, it would follow, since $S$ has $d\left(w_{1}\right)$ elements with degrees not exceeding $d\left(w_{1}\right)$, that $S \cup\left\{w_{m+1}\right\}$ was a set of $\frac{1}{2}(n+1)$ vertices with degrees not exceeding $\frac{1}{2}(n-1)$, which is precluded by (ii). It follows that $d\left(w_{1}\right)+d\left(w_{m+1}\right) \geqq n$. Therefore $w_{m+1}$ is nonadjacent to at most $d\left(w_{1}\right)$ vertices, and, since $w_{m+1}$ itself is one of these, the $d\left(w_{1}\right)$ elements of $S$ include a vertex $w_{i}$ adjacent to $w_{m+1}$.

For this value of $i$, let $X$ be the way with vertex sequence (1). We note that $x_{1}=w_{i}$ is adjacent to $x_{m+1}=w_{m+1}$. Then, if $m$ were less than $n-1$, the connectedness of $G$ would imply that some vertex $v$ not in $X$ was adjacent to a term $x_{j}$ of $X$, which is impossible since in this event

$$
v, x_{j}, x_{j+1}, \cdots, x_{m+1}, x_{1}, x_{2}, \cdots, x_{j-1}
$$

would be a way of length $m+1$. Therefore $m=n-1$ and $X$, together with the edge joining $x_{1}$ to $x_{m+1}$, contributes a Hamiltonian circuit of $G$.

I am indebted to Professor F. Harary for suggesting several improvements in this paper.

\section{REFERENCE}

1. L. Posa, A theorem concerning Hamiltonian lines, Magyar Tud. Akad. Mat. Kutató Int. Közl. 7 (1962), 225-226.

University of Waterloo, Waterloo, Ontario, Canada and

University of AbERDEEN, SCOTLAND 